\title{
The Superposition Principle: A Conceptual Perspective on Pedestrian Stream Simulations
}

\author{
Michael J. Seitz ${ }^{1,2} \cdot$ Felix Dietrich $^{1,2} \cdot$ Gerta Köster $^{1}$. \\ Hans-Joachim Bungartz ${ }^{2}$ \\ ${ }^{1}$ Munich University of Applied Sciences, Munich, Germany, \\ E-mail:m.seitz@hm.edu \\ 2 Technische Universität München, Garching, Germany
}

Received: 5 August 2015 / Accepted: 28 November 2015

DOI: $10.17815 / C D .2016 .2$

\begin{abstract}
Models using a superposition of scalar fields for navigation are prevalent in microscopic pedestrian stream simulations. However, classifications, differences, and similarities of models are not clear at the conceptual level of navigation mechanisms. In this paper, we describe the superposition of scalar fields as an approach to microscopic crowd modelling and corresponding motion schemes. We use this background discussion to focus on the similarities and differences of models, and find that many models make use of similar mechanisms for the navigation of virtual agents. In some cases, the differences between models can be reduced to differences between discretisation schemes. The interpretation of scalar fields varies across models, but most of the time this variation does not have a large impact on simulation outcomes. The conceptual analysis of different models of pedestrian dynamics allows for a better understanding of their capabilities and limitations and may lead to better model development and validation.
\end{abstract}

Keywords Crowd models · simulation · microscopic $\cdot$ scalar fields $\cdot$ superposition · social forces $\cdot$ local optimisation $\cdot$ pedestrians $\cdot$ discretisation $\cdot$ comparison of models

\section{Introduction}

Pedestrian and crowd dynamics are increasingly being studied using microscopic simulation models [1]. In engineering, simulation studies predicting the movement of crowds can help to improve the safety and comfort of pedestrians. Furthermore, the simulation 

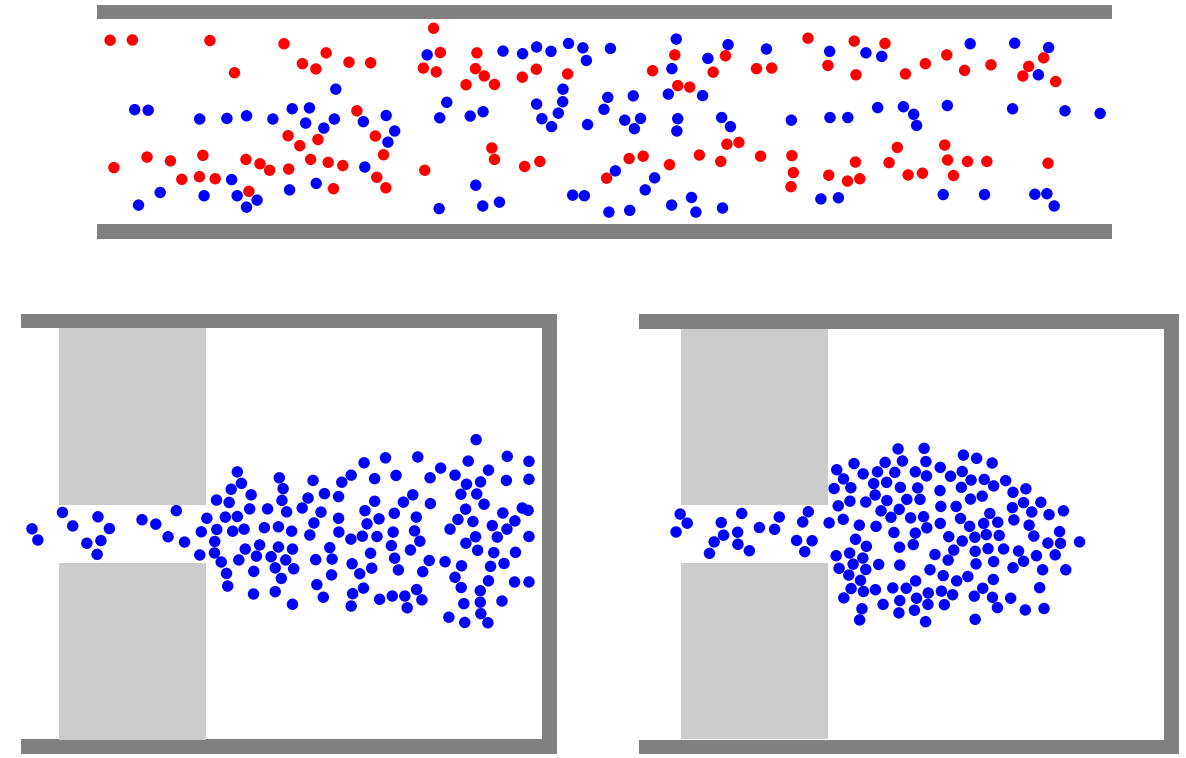

Figure 1 Examples of simulation scenarios illustrating how simulations are used to investigate crowd phenomena. In the upper figure, distinct lanes are formed by pedestrians streams in two different directions (blue from right to left, red from left to right). In the two figures at the bottom, pedestrians walk from right to left passing through a bottleneck. Both the left and right figure show the same scenario at different times. In the left figure, pedestrians have started to pass through the bottleneck and a congestion starts to form in front of it. In the right figure, all pedestrians have reached the congestion in front of the bottleneck or have already passed through it.

models themselves can be used to study the dynamics of pedestrian streams and thus serve as a means to scientific inquiry [2]. Simulated pedestrians (hereafter referred to as agents) are highly abstracted and simplified in their physical representation and decision-making processes. Several phenomena observed in experiments or field observations have been reproduced or predicted, such as the formation of lanes or congestions at bottlenecks [3] (see Fig. 1).

A variety of models that predict pedestrian behaviour have been proposed (for reviews see [4-6]). Two approaches stand out due to their widespread use: cellular automata [7-10] and force-based models [3,11-13]. At first glance, these approaches seem to have little in common. For example, force-based models are continuous in both time and space; cellular automata are discrete in both time and space. While in force-based models motion is determined through the manipulation of the acceleration, in cellular automata, the next position of agents is determined directly leading to a discrete motion process.

In this paper we argue that most models have one commonality: navigation decisions are based on evaluating scalar fields. A scalar function is a function with one or more inputs and a one-dimensional output. When evaluated on the whole plane, scalar functions generate scalar fields (sometimes referred to as floor fields). In the models we discuss, this scalar field is a superposition of other scalar fields representing individual contributions 
to the overall behaviour of agents, such as keeping a certain distance from one another and approaching a target. The scalar field is, for example, interpreted as potential that accelerates agents in force-based models or the probability of agents moving to an adjacent cell in cellular automata. Although scalar fields are a broad concept, they can be useful to analyse models on a conceptual level: even cellular automata and force-based models can be found to have several key features in common.

With the perspective of scalar fields, we aim to identify commonalities and differences among models that use the superposition of scalar fields. If the aspects in which the models differ are characterised, a new useful classification of the models can be undertaken. Key aspects in which the models differ are space and time discretisation, and whether they model the position, velocity, or acceleration explicitly. Based on this characterisation, we identify conceptual strengths and challenges in the various approaches such as force-based models, cellular automata, the gradient navigation model, and the optimal steps model. Models that do not use the superposition of scalar fields as a basic concept are not included in our analysis. However, the scalar field perspective helps to identify and classify the models that use a fundamentally different approach. We discuss how the perspective may contribute to future model development and analysis.

The paper is structured as follows. First, we outline individual-based modelling for pedestrian dynamics. We set the formal frame for dynamical systems and scalar fields and explain known relevant simulation approaches. Second, we present models and compare them with respect to various characteristics. Finally, we discuss implications and future directions of the results.

\section{Pedestrian motion based on the superposition of scalar fields}

In this section, we first give a descriptive explanation of several models for pedestrian simulation. Then, we develop a formal perspective on these models: the superposition of scalar fields that yields another scalar field. Finally, we describe how the scalar field is interpreted in the different models. All of the approaches describe pedestrian motion in two dimensions in the transverse plane.

\subsection{Modelling approaches}

The two modelling approaches most commonly used for the simulation of individual pedestrians are force-based models and cellular automata. Both have been described in the literature on pedestrian simulation with a variety of extensions and modifications. Their basic principles are outlined in this section.

The social force model was first presented by Helbing and Molnár [3] in an attempt to capture qualitative features such as lane formation and congestion at bottlenecks. The basic idea is that pedestrian behaviour can be simulated with mathematical equations that describe forces acting on the agents (see Fig. 2(a) and 3(a)). These forces lead to 


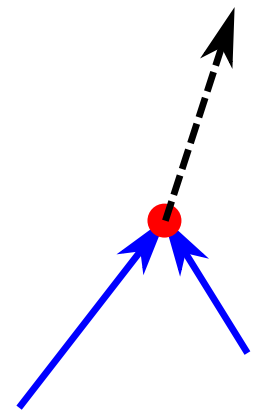

(a)

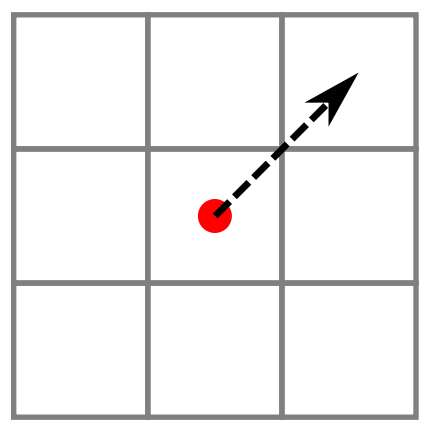

(b)

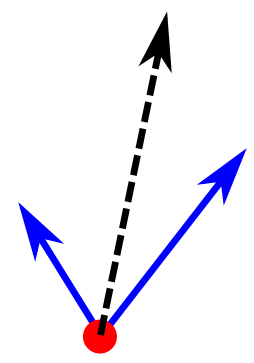

(c)

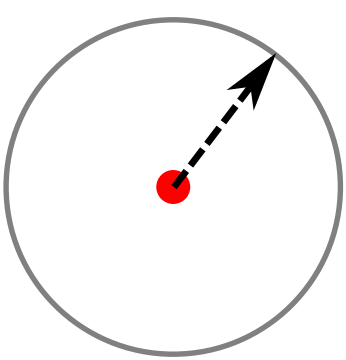

(d)

Figure 2 Locomotion schemes of four pedestrian stream simulation models. (a) Social force model: agents are accelerated by forces acting on them as in particle physics. (b) Cellular automaton: agents occupy one cell and move forward by moving to an adjacent cell. (c) Gradient navigation model: the direction of movement is determined directly and continuously in time. (d) optimal steps model: Agents move forward in a stepwise manner, but place the next step locally around the current position in continuous space. The radius of the reachable circle coincides with the step length of real pedestrians.

acceleration in various directions, such as in the direction of the target or away from other agents. Hence, the forces are evaluated as in physics, and lead to similar emergent effects, such as inertia, which seems plausible for pedestrians to a certain degree. Later, behavioural features were added to reproduce "escape panic" [11], although the concept of "panic" is highly questionable [14-16]. Further extension and modifications have been proposed for the original idea of social forces [13,17,18]. Many of these modifications try to mitigate the effects introduced by the physical nature, such as oscillations or inertia [12].

More or less independently of force-based models, several approaches based on cellular automata have been proposed [7-10,20-22]. The first one published known to the authors is the model by Gipps and Marksjö [7]. In cellular automata, the transverse plane is divided into a grid of cells that are either rectangular, hexagonal, or, in theory, triangular (see Fig. 2(b), 3(b), and 5). In most models, agents occupy one cell on this grid. However, there are also models that have a finer cellular grid with pedestrians occupying more than one cell [22]. The agents move forward by jumping to one of the adjacent cells. The decision of where to move next is made by either choosing the best cell according to some criterion [7], by drawing from a probability distribution describing the likelihood of the agent's move to each of the adjacent cells [10], or according to a set of simple decision rules $[8,9,20]$. The last category cannot be explained well with scalar fields, and hence we do not include it in our analysis. If a cell can only be occupied by one agent, the size of the cell also determines the simulated pedestrian's size. Furthermore, the movement step length of simulated pedestrians is the distance from one cell's centre to the centre of the next cell. However, the size of cells can be reduced, which changes the model's behaviour [23].

In both force-based models and cellular automata, some issues exist that have to be 


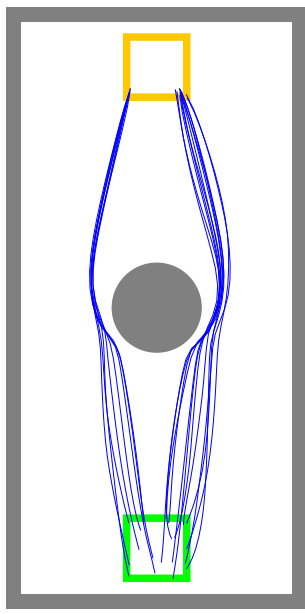

(a)

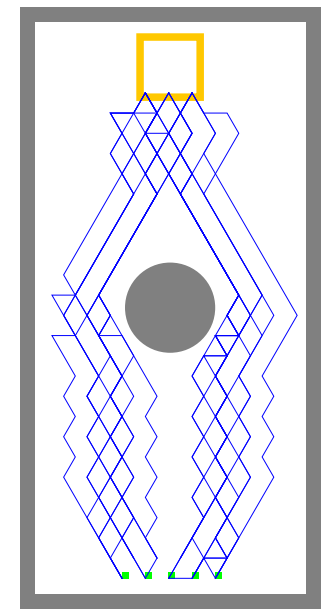

(b)

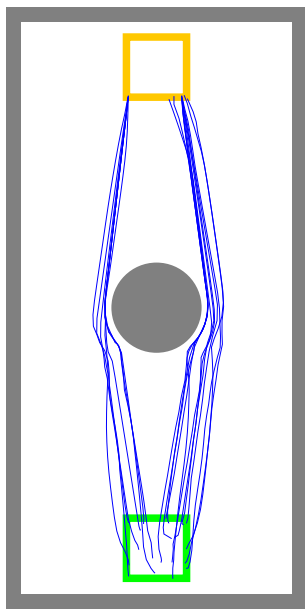

(c)

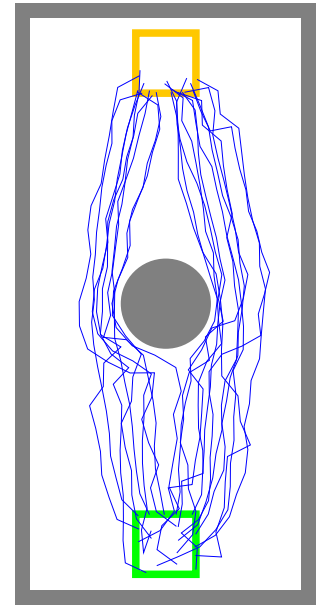

(d)

Figure 3 Emergent trajectories of different pedestrian simulation models: (a) the social force model, (b) a cellular automaton with a hexagonal grid, (c) the gradient navigation model, and (d) the optimal steps model. 20 agents move from the bottom green rectangle to the upper yellow rectangle (the agents were placed on the grid points for the cellular automaton). On their way, the agents encounter an obstacle in the form of a column, which they have to skirt. The social force model (a) and the gradient navigation model (c) both produce smooth trajectories. In the cellular automaton and the optimal steps model, agents make discrete motion steps. With the cellular automaton (b), the possible directions of motion are visible. In contrast, agents in the optimal steps model (d) can move in all directions. (For more details on the comparison of models, see Fig. 2 and [19]).

dealt with in order to produce realistic pedestrian trajectories. For instance, in social force models, inertia may lead to a bouncing effect, and in cellular automata, the grid structure may lead to an undesired preference of some movement directions. Two alternative modelling approaches that try to eliminate such artefacts are the gradient navigation model [24] and the optimal steps model [25-27]. Both of these models follow a different approach compared to force-based models and cellular automata but also show some similarities (see [19] for a comparison of emergent behaviour). The gradient navigation model directly manipulates the motion direction in continuous space and time (see Fig. 2(c) and 3(c)). Thus, in contrast to force-based models, the direction is determined with the first derivative, that is, directly through the speed of motion. In force-based models, the second derivative, the acceleration, is manipulated.

The optimal steps model produces stepwise movement as do cellular automata. However, in the optimal steps model, this step is taken in continuous space and time [25,27]. Hence, the stepping process is not continuous itself, but the time when the step is taken and the position it is taken to are in continuous space (see Fig. 2(d) and 3(d)). The position of the next step is determined through optimisation as is the case in some cellular automata. In contrast to cellular automata, in the optimal steps model, there is no grid structure and the step length is that of a pedestrian measured empirically. Hence, one does not have to deal with grid artefacts and other challenges that arise in cellular au- 
tomata due to the cell structure.

There are alternative models that cannot be described well with the superposition of scalar fields. One approach that is well known in game development is steering behaviours [28]. Here, a variety of behaviours are represented by vectors pointing in possibly different directions. Although this may seem similar to the gradient navigation model and social force model, the vectors are modelled and interpreted directly and are not the product of a scalar field. Other examples that cannot be described well with the superposition principle are velocity-obstacle models [29], which were originally developed in robotics. The underlying assumption of velocity-obstacle models models is that agents choose trajectories without collisions. The velocity of agents is chosen by considering the positions and velocities of other agents and obstacles. A more recent model is based on simple rules that determine the motion process [30]. Here, direction and speed are computed through optimisation of the time to the next collision. Although the underlying locomotion is based on the social force model, the actual decision-making process is not based on the superposition of scalar fields in the sense we define below. Finally, a concept that is currently being studied for pedestrian dynamics is represented by optimal velocity models, which stem from traffic simulations [31,32].

\subsection{A formal perspective}

All models in pedestrian dynamics describe alterations to a given state $x$ over a certain time interval. In most microscopic approaches, this state contains variables of all individuals, such as their positions, velocities, or current targets.

Models in pedestrian dynamics can be classified in a very abstract way, using terminology from dynamical systems theory $[33,34]$ in mathematics. A model, according to our terminology here, is used to describe and reproduce certain aspects of reality, and a system is a combination of a model and a state. For example, a differential equation with a given initial state is a system. The equation without the initial state can then be interpreted as the model of some natural phenomenon, but the initial state is not part of the model.

In the following, we focus on two classes containing all pedestrian simulation models. In the first class, $x_{n}$ is the current state of the system and $n \in \mathbb{N}$ is the discrete time step. A function $f$ is the core of the system and uses the current state and time information to advance the state. The class contains discrete systems of the form

$$
x_{n+1}=f\left(n, x_{n}\right) .
$$

In the second class, $x(t)$ is the current state and $t \in \mathbb{R}$ is the current time. The class is comprised of continuous systems of the form

$$
\dot{x}(t)=f(t, x(t)),
$$

where $\dot{x}$ is the time derivative of the state $x$. Hence, Eq. 2 is a differential equation.

In microscopic models for pedestrian dynamics, the function $f$ is usually decomposable into an array of functions $f_{i}$, one for each of the $N$ agents:

$$
f=\left(f_{1}, f_{2}, \ldots, f_{N}\right) .
$$


In most models, all functions $f_{i}$ have the same form and only vary in the values of their parameters, such as free-flow speed or the desired target. This can be an advantage: because all functions have the same form, the modelling only has to be done once on the individual level, not separately for each agent. Individual modelling would be necessary in a more heterogeneous environment, for example with cars and public transport.

Each function $f_{i}$ uses a subset of the information contained in the current state $x$ to advance the state of agent $i$. For instance, only the positions of the neighbours of agent $i$ are used to update the position of agent $i$. All models under consideration in this paper first transform the information contained in the state $x$ with a superposition of scalar functions before it is used by $f_{i}$ to advance the state. In the following, we address this property.

Scalar functions are used and interpreted in many different ways in pedestrian simulation models. One way is to yield the distance to the target, given any position in twodimensional space. In this case, there are two inputs (the $x$ - and $y$-coordinate), and the output is the shortest distance to the target from the given position. This distance need not be the direct Euclidean distance to the target: for pedestrians in a scenario with obstacles on the way to the target, the shortest path to the target must lead around the obstacles. The path can be computed using visibility graphs [35,36], Dijkstra's algorithm [35,37] or the Fast Marching algorithm [38-41].

Mathematically, a scalar function $s$ is a function with $n$ inputs $\theta_{1}, \ldots, \theta_{n}$ and one real output:

$$
\begin{aligned}
s: \Theta^{1} \times \Theta^{2} \times \cdots \times \Theta^{n} & \rightarrow \mathbb{R} \\
\left(\theta_{1}, \theta_{2}, \ldots, \theta_{n}\right) & \mapsto s\left(\theta_{1}, \theta_{2}, \ldots, \theta_{n}\right),
\end{aligned}
$$

where $\Theta^{i}$ is the $i$-th input space, for example the position space $\mathbb{R}^{2}$ of an agent. A scalar function $s$ can be used to generate a scalar field when applied to a whole set of inputs (see Fig. 4). This is the mathematical background for the so-called navigation fields [40, 42], most commonly used for cellular automata. Models based on differential equations, such as the social force model, often use the derivative of the scalar field. This new field comprises the scalar field's gradients and hence is a vector field.

In some models, the scalar functions only depend on the distance between agents or to a target and decays rapidly when the distance increases. However, many models in pedestrian dynamics also use information such as a field of vision or the current velocity of neighbours as inputs to their scalar functions.

Advantages of using a superposition of scalar fields mostly stem from the simplicity and assumed linear independence of their combination in a sum. Usually, the scalar fields induced by individual agents all have the same form, which facilitates modelling and analysis. However, the simplicity of the superposition also introduces certain challenges. Local minima in potential surfaces sometimes unrealistically trap agents, for example at bottlenecks in front of narrow doors. It is also not clear whether humans assess all cues in their surrounding independently. Moussaïd et al. [30] discuss the problem of combining binary interactions to one movement decision. To summarise, the superposition of scalar fields seems to be a useful tool in modelling spatial agent interactions and dynamics. However, one has to be careful in their construction since a combination - even of simple parts - can lead to very complex emergent effects. 


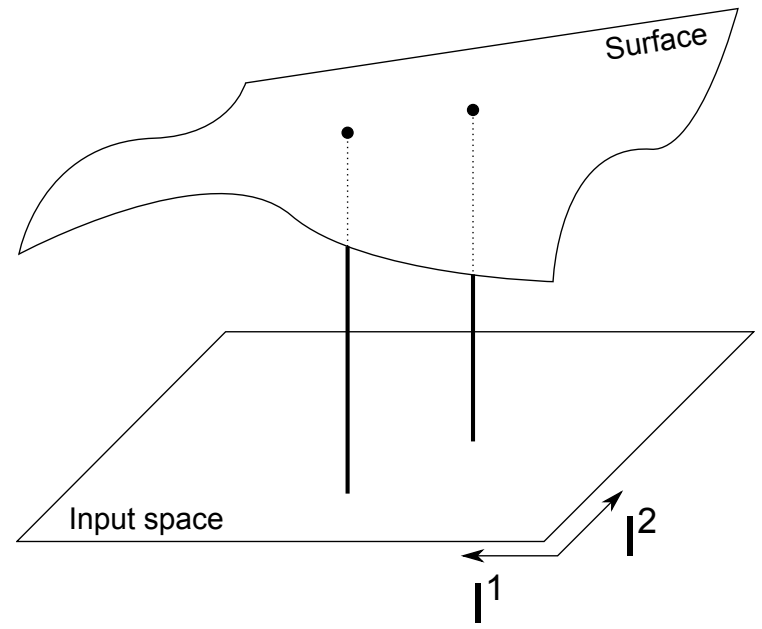

Figure 4 Scalar function represented as surface, which is a scalar field over the input space. The input space is $I^{1} \times I^{2}$, and the scalar field is the image of the input space through a scalar function $s$. Two positions evaluated by $s$ are depicted as lines ending in points on the surface. The scalar function $s$ only yields the height of the two points over the $I^{1}-I^{2}$-plane, not their $I^{1}$ or $I^{2}$ coordinates.

\subsection{Interpretation of the scalar field}

In this section we describe how models use the scalar field as they were defined in the previous section. In addition to the mathematical use of the scalar field, we also discuss its functional interpretation. Our discussion encompasses mainly four types of interpretations: utility, probability density, potential, and velocity. This discussion illustrates how the various approaches use scalar fields and prepares for the following section, which is dedicated to the comparison of models.

In cellular automata, one scalar value is assigned to each cell. This can be interpreted as all positions within a cell having the same value, positions other than the centre being ignored, or the scalar function being only defined for the centre of the cell. In practice, only one value has to be stored for each cell. An agent in the simulation evaluates the adjacent cells and makes a decision based on their values. In probabilistic cellular automata [10], the scalar field is interpreted as a probability density for the move to the next cell. Thus, the sum of the values of all adjacent cells has to be 1. This includes a certain probability for the cell the agent is currently occupying, reflecting the probability that the agent will remain at the current position. In deterministic cellular automata, each cell can have an arbitrary value. Agents optimise the next position by choosing the cell with the highest or lowest value [7]. In both cases, the values have to be corrected for the distance to that cell. This is important when the adjacent cells are not equally far away from each other, as is the case in cellular automata with rectangular grid and Moore neighbourhood. When choosing the highest value, the scalar field can be interpreted as utility which is maximised. If the smallest value is chosen, the scalar field can be interpreted as potential which is minimised. However, the latter interpretation may be misleading, since the model does not actually compute forces, which the word "potential" might suggest. 
In the optimal steps model $[25,27]$, the same interpretations as for cellular automata are possible. However, the actual computation is different. Since for the optimal steps model the plane is not divided into cells, optimisation takes place in continuous space. For each step, a circle is placed around the current position of the agent. The radius of the circle coincides with the step length of a real pedestrian in a situation where the pedestrian can move without obstruction. Then the next position is chosen either on the circle or the disc, which leads to a one-dimensional or two-dimensional optimisation problem, respectively. Hence, the scalar field must be defined for all positions in the plane, not only for the centre of cells. Alternatively, the scalar field could be interpreted as a probability density distribution using a step circle. This would yield a new probabilistic model similar to probabilistic cellular automata but without the cell structure.

The scalar field in the gradient navigation model [19] is best interpreted as utility ${ }^{1}$. However, the optimisation of the utility function is not explicit as in cellular automata and the optimal steps model. Instead, the direction of motion and the speed (the velocity) are chosen according to the gradient at the current position of the agent. More specifically, the direction is determined directly with the first derivative of the position leading to a continuous motion process, and the derivative is modelled with the negative gradient of the scalar field. However, in a practical formulation of the model, the gradient is specified and computed directly and not through the derivative of the scalar field.

Finally, in force-based models $[3,11-13,17]$, the scalar field is interpreted as potential leading to forces and finally to acceleration. The acceleration is integrated to velocity, which is again integrated to obtain the current position. The acceleration is modelled through the second derivative of the position leading to a continuous motion process. Again, in practical formulations, the potential is not modelled explicitly but rather the forces, which are represented by vectors.

Hence, in both force-based models and the gradient navigation model the scalar field is an underlying modelling idea that is not necessarily visible in the formulations. Conceptionally, the interpretations are different for the two approaches: in the gradient navigation model, they determine the velocity, and in force-based models, they determine the acceleration. In general, since the input to the scalar functions is not restricted, it can be used to model any kind of behaviour when combined with the interpretations above. However, vector valued functions can be more concise than scalar functions for certain models, such as force-based models and the gradient navigation model. For these models, the gradients can be constructed directly from the positions of other agents instead of constructing a scalar field first and then computing the derivative.

\section{Comparison of modelling concepts}

In this section, we investigate similarities and differences among the models described above. There are three key aspects of models that may differ to a certain degree: space and time discretisation, numerics, and emergent phenomena.

\footnotetext{
${ }^{1}$ In the original publication [24] the values were minimised and thus could be interpreted as having negative utility or potential.
} 


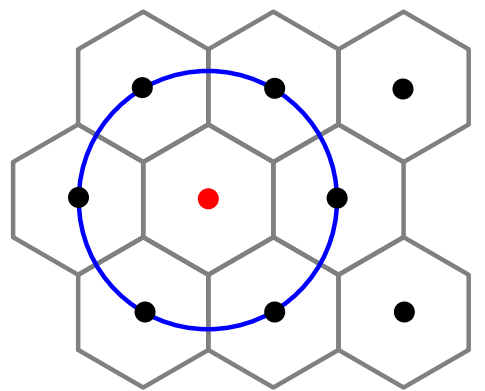

(a)

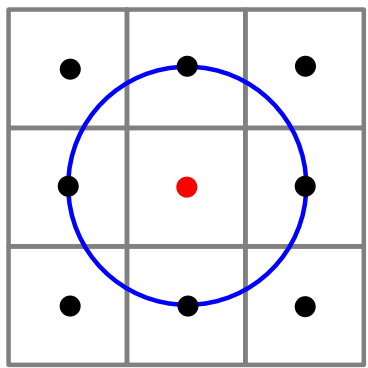

(b)

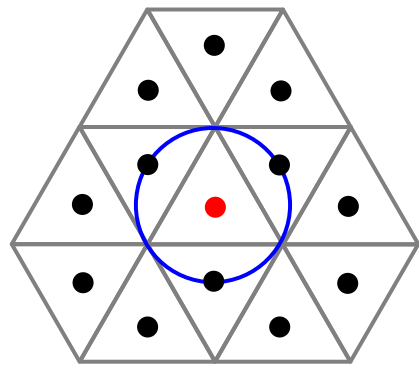

(c)

Figure 5 Possible grid types in cellular automata: (a) hexagonal, (b) rectangular, and (c) triangular. A hexagonal lattice may be preferable because of its geometrical correspondence to close-packed equal spheres [25], the equal distance to all adjacent cells, and the equal angle between all adjacent possible directions. Locomotion in cellular automata can be emulated by the optimal steps model if the step circle and discretisation are chosen in a specific way as is indicated by the blue circles.

\subsection{Discretisation and numerics}

Space discretisation is one of the most important features of every pedestrian motion simulation and can be part of the model formulation or the numerical solution. In cellular automata, the space discretisation is determined by the lattice, that is, the shape and size of grid cells (see Fig. 5). The minimal distance agents can move forward in cellular automata is the distance from the current cell to an adjacent cell. Hence, the space discretisation is an integral part of the model. Space discretisation also affects time discretisation: to obtain faster speeds, agents either have to be allowed to move forward more than one cell, or time steps have to be reduced [23,43].

In the optimal steps model, locomotion is stepwise as in cellular automata, and the next step can be taken to an arbitrary position on the step circle or disk. Thus, movement is not bound to a lattice and is discrete but in continuous space. Movement is not, however, continuous as in force-based models and the gradient navigation model. Movement of agents in cellular automata can be emulated if the numerical solution for the optimisation is chosen in a specific way [25]. When the next position is chosen on the whole disk, smaller steps are possible allowing for smoother movement and stream behaviour [44].

In the following, we show how the optimal steps model can be modified to converge towards the gradient navigation model. For this, we introduce another approach, the gradient steps model, which is a combination of the optimal steps model and the gradient navigation model. The gradient steps model is an example that demonstrates how the conceptual considerations developed in this paper can be useful to close gaps between models. In this example, the stepwise movement of the optimal steps model is preserved, but no explicit optimisation is conducted. Instead, the direction is determined directly through the gradient as in the gradient navigation model. The speed can be manipulated in the same way as in the optimal steps model $[25,26]$. Since only the local position of the agent is evaluated for the gradient, overlapping of pedestrians cannot be avoided in 


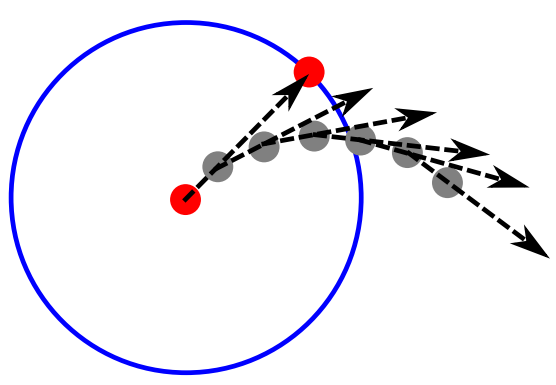

Figure 6 Illustration of the motion schemes in the gradient navigation model and the gradient steps model. The blue circle represents the step circle. The arrows indicate the gradient at the respective positions. The gray dots are possible positions in the gradient navigation model, and the red dots are possible positions in the gradient steps model. This comparison illustrates how agents assume different positions given the same scalar field but with another discretisation.

the same way as in the optimal steps model. Furthermore, due to the limited information being used, the emergent dynamics are different to those of the optimal steps model and rather resemble those of the gradient navigation model.

In the gradient navigation model, discretisation is a numerical issue while solving the ordinary differential equations resulting from the model's formulation. For this, very small steps are made by agents to simulate a continuous motion process described by the underlying ordinary differential equation. The speed and direction are computed again for each of those steps. If larger steps were taken, the locomotion would result in a discrete motion process. Hence, if steps are taken as long as those in the gradient steps model, the two models converge.

Eq. 6 to 8 state this convergence in a formal way. The differential setting, as in forcebased models and the gradient navigation model, is given by

$$
\frac{d x}{d t}=\dot{x}=f(x) .
$$

If time is discretised with time step $\Delta t$, the differential is reformulated as a difference:

$$
\begin{aligned}
\frac{x(t+\Delta t)-x(t)}{\Delta t} & =f(x(t)), \\
\Rightarrow x(t+\Delta t) & =x(t)+\Delta t \times f(x(t)) .
\end{aligned}
$$

The discretisation can be used to solve the model in Eq. 6 numerically. When the time step is chosen large (e.g. $0.5 \mathrm{~s}$ ), formulation 8 is a discrete model, such as a cellular automaton or the optimal steps model. Although the function $f$ usually varies with different model formulations - whether continuous or discrete - it can be chosen to be identical for some models. Then, only the time step length $\Delta t$ would differ, and the models converge if $\Delta t$ is set to the same value for both models. For the numerical solution of differential equations, however, the time step cannot be chosen arbitrarily large. 
Force-based models have no intrinsic space discretisation in their formulation and thus are continuous. As in the gradient navigation model, discretisation is an issue of numerically solving the ordinary differential equations resulting from the model's formulation (also see [45]). Since the velocity is only manipulated indirectly through the acceleration, force-based models can be seen as principally different to the other models. However, if larger locomotion steps were taken by agents, a discretisation could be chosen that resembles the stepwise motion of the gradient and optimal steps model.

Time and space discretisation are strongly related in force-based models and the gradient navigation model: if a larger time step is chosen, the motion step also increases. Hence, the choice of time step length concerns numerical accuracy and not the model itself. In cellular automata and the optimal steps model, the time step is a model parameter. The longer the simulation time step, the more agents move at the same time. This approach is called time-slicing. Alternatively, agents can be allowed to move at an arbitrary point in time when the last step is finished according to the model [26]. This is called an event-driven scheme or discrete-event simulation (e.g., see [46] for a general treatment in simulation theory).

\subsection{Assessment of modelling concepts}

We assess features of the models in this section. Some features are directly part of the model; others follow from its mechanisms. From a conceptual modelling perspective, there are some crucial differences in the interpretation of the scalar field.

Force-based models are based on an analogy from physics. The scalar field is interpreted as potential and then used as such for the physical motion of agents almost exactly in the way the motion of particles would be computed. On the one hand, forces resulting from potentials are interpreted as "social forces" [3], which is a concept previously used in sociology [47]. On the other hand, social forces are then used directly as physical forces to compute agents' acceleration. Conceptionally it seems questionable whether social forces, if we assume they exist, directly translate to physical forces. Using the analogy of physical forces has been shown to be a seminal approach for the simulation of pedestrian dynamics $[11,12]$.

In contrast to force-based models, probabilistic cellular automata describe pedestrians' movement from a rather phenomenological perspective [48]. Here, the behaviour of pedestrians is not explained in detail because only a certain probability is assigned to a specific outcome. However, the scalar field as probability density function can still be interpreted as the pedestrian's motivation or probabilistic decision-making process.

In deterministic cellular automata, the scalar field can be interpreted as utility, which can then be used in an optimisation scheme. Although the idea of utility optimisation has been questioned for human decision making [49], conceptionally, it is a coherent concept. The same is true for the optimal steps model, which explicitly optimises utility for the next step. Here, no analogy is needed since utility optimisation stands for itself as a decision-making process. As mentioned previously, the gradient navigation model can also be interpreted as indirectly optimising utility, and hence the scalar field is best interpreted as utility. 
Mathematically, there are three major model classes: ordinary differential equations, optimisation, and probabilistic dynamics. Force-based models and the gradient navigation model build on ordinary differential equations since they are formulated as the first or second derivative of agents' positions, which can lead to numerical challenges that have to be dealt with [45]. In deterministic cellular automata and the optimal steps model, a numerical optimisation scheme has to be employed. For cellular automata, the values of the adjacent cells simply have to be compared. In the optimal steps model, a similar approach can be taken with placing a grid on the circle or disc $[25,27]$, or, alternatively, employing a numerical optimisation scheme [44]. In probabilistic cellular automata, a random variable has to be drawn from the probability distribution, which is constructed based on the scalar field.

The different features of the various models allow for different perspectives on emergent effects or phenomena. Force-based models can be used to incorporate contact forces [11], which is an important aspect in crowds with very high densities. Furthermore, agents move in continuous space, which yields a smooth trajectory and a detailed perspective on an agent's motion process. The gradient navigation model also simulates continuous locomotion. Although the scalar field does not yield forces, the motivation is modelled more directly through the direction of motion and thus may allow for better control of emergent behaviour. Cellular automata facilitate fast computation due to the implicit data structure given by the cellular grid and smaller number of function evaluations. In the optimal steps model, locomotion is not continuous but rather in continuous space, thus allowing for a free motion process. New opportunities to study pedestrians' real stepping behaviour may result from this modelling approach $[27,44]$.

\subsection{Implications for model development}

Models can be studied from different perspectives, such as their predictions and emergent effects, their mathematical and numeric properties, and their conceptional structure. Understanding the conceptual differences in modelling approaches is essential for model development. Most importantly, the conceptual perspective helps to make modelling decisions because knowing whether certain model features are available may be necessary for a specific problem.

Certain limitations of the superposition principle are inherent to all models based on it. For instance, it may not always be possible to calibrate the parameters to obtain meaningful emergent behaviour for different scenarios [50]. The introduction of psychological or physiological aspects, such as a field of vision, can lead to discontinuities in the scalar field over time. These discontinuities can be a problem for models based on differential equations and some optimisation schemes [44,45]. On the other hand, extensions developed for one model based on scalar fields can easily be adopted for other models within the superposition paradigm. For example, sub-group behaviours $[18,51]$ and navigation fields $[40,42]$ were developed for force-based models and cellular automata, respectively, but were successfully used subsequently in other models afterwards.

New models can result from insights into the limitations of the superposition principle. For instance, it is not always clear how to combine the binary interactions captured with 
individual scalar fields [30]. It is also unlikely that humans integrate the positions of all other pedestrians around them into one compromise decision, but rather use heuristic information gathering and decision making $[49,52]$. We propose that alternative models also aim at the cognitive process rather than just at observable behaviour [30,50].

\section{Conclusions}

In this paper we provided a conceptual perspective on microscopic modelling approaches to pedestrian dynamics. We described how several models use a scalar field as a common core feature. The scalar field itself is then interpreted in different ways as potential, probability distribution, or utility. Several model differences result from this interpretation, such as the discretisation of space and time. We described how the models converge if certain parameters are chosen in a specific way. The assessment of modelling concepts led us to implications of our perspective on pedestrian simulation models. Finally, based on this, we proposed directions for future model development.

Usually, pedestrian simulation models are assessed by an aggregated measure, such as the density-flow relation, or sometimes, by the agents' individual emergent behaviour. Here, we studied the conceptual perspective of scalar fields focusing on model similarities. The perspective helps to assess the different approaches and guide future model development.

Scalar fields form the basis for a variety of models that capture crowd phenomena with a concise mathematical or algorithmic description. Therefore, it seems likely that this approach will remain useful for a number of applications and scenarios in the future. For example, fast computations can be realised with cellular automata and high crowd densities are well captured by force-based models. However, for a fundamental understanding of collective pedestrian behaviour, new approaches and paradigms should be explored. Promising directions are optimal velocity models stemming from traffic simulations, models that try to reproduce the cognitive process, and more specifically, cognitive heuristics.

In order to select a model - whether it is based on the superposition principle or not - similarities and differences have to be known on a conceptional level. Therefore, the perspective proposed here may lead to a better classification of existing models, inform the decision on which model to use, facilitate the exchange of concepts within the paradigm, and inspire new model developments beyond it.

\section{Acknowledgements}

This work was partially funded by the German Federal Ministry of Education and Research through the project MultikOSi on assistance systems for urban events - multicriteria integration for openness and safety (Grant No. 13N12824). The authors gratefully acknowledge the support by the Faculty Graduate Center CeDoSIA of TUM Graduate School at Technische Universität München, Germany. Support from the TopMath Grad- 
uate Center of TUM Graduate School at Technische Universität München, Germany, and from the TopMath Program at the Elite Network of Bavaria is gratefully acknowledged.

\section{References}

[1] Daamen, W., Duives, D.C., Hoogendoorn, S.P. (eds.): The Conference in Pedestrian and Evacuation Dynamics 2014 (PED 2014), Transportation Research Procedia, Pages 1-818, vol. 2. Elsevier, Delft, The Netherlands (2014). URL http: / / www . sciencedirect.com/science/journal/23521465/2/

[2] Strube, G.: Generative theories in cognitive psychology. Theory \& Psychology 10(1), 117-125 (2000). doi:10.1177/0959354300010001606

[3] Helbing, D., Molnár, P.: Social Force Model for pedestrian dynamics. Physical Review E 51(5), 4282-4286 (1995). doi:10.1103/PhysRevE.51.4282

[4] Zheng, X., Zhong, T., Liu, M.: Modeling crowd evacuation of a building based on seven methodological approaches. Building and Environment 44(3), 437-445 (2009). doi:10.1016/j.buildenv.2008.04.002

[5] Papadimitriou, E., Yannis, G., Golias, J.: A critical assessment of pedestrian behaviour models. Transportation Research Part F: Traffic Psychology and Behaviour 12(3), 242-255 (2009). doi:10.1016/j.trf.2008.12.004

[6] Duives, D.C., Daamen, W., Hoogendoorn, S.P.: State-of-the-art crowd motion simulation models. Transportation Research Part C: Emerging Technologies 37(0), 193 209 (2013). doi:10.1016/j.trc.2013.02.005

[7] Gipps, P., Marksjö, B.: A micro-simulation model for pedestrian flows. Mathematics and Computers in Simulation 27(2-3), 95-105 (1985). doi:10.1016/0378-4754(85)90027-8

[8] Blue, V.J., Embrechts, M.J., Adler, J.L.: Cellular automata modeling of pedestrian movements. In: IEEE International Conference on Systems, Man, and Cybernetics, pp. 2320-2323 (1997)

[9] Blue, V.J., Adler, J.L.: Cellular automata microsimulation for modeling bidirectional pedestrian walkways. Transportation Research Part B: Methodological 35, 293-312 (2001). doi:10.1016/S0191-2615(99)00052-1

[10] Burstedde, C., Klauck, K., Schadschneider, A., Zittartz, J.: Simulation of pedestrian dynamics using a two-dimensional cellular automaton. Physica A: Statistical Mechanics and its Applications 295, 507-525 (2001). doi:10.1016/s0378-4371(01)00141-8

[11] Helbing, D., Farkas, I., Vicsek, T.: Simulating dynamical features of escape panic. Nature 407, 487-490 (2000). doi : 10 . $1038 / 35035023$ 
[12] Chraibi, M., Kemloh, U., Schadschneider, A., Seyfried, A.: Force-based models of pedestrian dynamics. Networks and Heterogeneous Media 6(3), 425-442 (2011). doi:10.3934/nhm.2011.6.425

[13] Chraibi, M., Seyfried, A., Schadschneider, A.: Generalized centrifugal-force model for pedestrian dynamics. Physical Review E 82(4), 046111 (2010). doi:10.1103/PhysRevE.82.046111

[14] Johnson, N.R.: Panic and the breakdown of social order: Popular myth, social theory, empirical evidence. Sociological Focus 20(3), 171-183 (1987). doi:10.1080/00380237.1987.10570950

[15] Aguirre, B.E.: Commentary on "understanding mass panic and other collective responses to threat and disaster": Emergency evacuations, panic, and social psychology. Psychiatry 68(2), 121-129 (2005). doi:10.1521/psyc.2005.68.2.121

[16] Drury, J., Novelli, D., Stott, C.: Representing crowd behaviour in emergency planning guidance: 'mass panic' or collective resilience? Resilience: International Policies, Practices and Discourses 1, 18-37 (2013). doi:10.1080/21693293.2013.765740

[17] Yu, W.J., Chen, R., Dong, L.Y., Dai, S.Q.: Centrifugal force model for pedestrian dynamics. Physical Review E 72, 026112 (2005). doi:10.1103/PhysRevE.72.026112

[18] Moussaïd, M., Perozo, N., Garnier, S., Helbing, D., Theraulaz, G.: The walking behaviour of pedestrian social groups and its impact on crowd dynamics. PLoS ONE 5(4), e10047 (2010). doi: 10.1371 / journal.pone. 0010047

[19] Dietrich, F., Köster, G., Seitz, M., von Sivers, I.: Bridging the gap: From cellular automata to differential equation models for pedestrian dynamics. Journal of Computational Science 5(5), 841-846 (2014). doi:10.1016/j.jocs.2014.06.005

[20] Fukui, M., Ishibashi, Y.: Self-organized phase transitions in cellular automaton models for pedestrians. Journal of the Physical Society of Japan 68(8), 2861-2863 (1999). doi:10.1143/JPSJ.68.2861

[21] Zhang, P., Jian, X.X., Wong, S.C., Choi, K.: Potential field cellular automata model for pedestrian flow. Physical Review E 85(2-1), 021119 (2012). doi:10.1103/PhysRevE.85.021119

[22] Was, J., Lubas, R.: Adapting social distances model for mass evacuation simulation. Journal of Cellular Automata 8, 395-405 (2013). Journal of Cellular Automata, Old City Publishing 
[23] Kirchner, A., Klüpfel, H., Nishinari, K., Schadschneider, A., Schreckenberg, M.: Discretization effects and the influence of walking speed in cellular automata models for pedestrian dynamics. Journal of Statistical Mechanics: Theory and Experiment 2004(10), P10011 (2004). doi:10.1088/1742-5468/2004/10/P10011

[24] Dietrich, F., Köster, G.: Gradient navigation model for pedestrian dynamics. Physical Review E 89(6), 062801 (2014). doi:10.1103/PhysRevE.89.062801

[25] Seitz, M.J., Köster, G.: Natural discretization of pedestrian movement in continuous space. Physical Review E 86(4), 046108 (2012). doi:10.1103/PhysRevE.86.046108

[26] Seitz, M.J., Köster, G.: How update schemes influence crowd simulations. Journal of Statistical Mechanics: Theory and Experiment 7, P07002 (2014). doi:10.1088/1742-5468/2014/07/P07002

[27] Seitz, M.J., Dietrich, F., Köster, G.: The effect of stepping on pedestrian trajectories. Physica A: Statistical Mechanics and its Applications 421, 594-604 (2015). doi:10.1016/j.physa.2014.11.064

[28] Reynolds, C.W.: Steering behaviors for autonomous characters. In: Game Developers Conference, pp. 763-782. Miller Freeman Game Group, San Francisco, California, San Jose, California (1999). URL http: / / www . red3d.com/cwr / papers/1999/gdc99steer.html

[29] Fiorini, P., Shiller, Z.: Motion planning in dynamic environments using velocity obstacles. The International Journal of Robotics Research 17(7), 760-772 (1998). doi:10.1177/027836499801700706

[30] Moussaïd, M., Helbing, D., Theraulaz, G.: How simple rules determine pedestrian behavior and crowd disasters. Proceedings of the National Academy of Sciences 108(17), 6884-6888 (2011). doi:10.1073/pnas.1016507108

[31] Tordeux, A., Seyfried, A.: Collision-free nonuniform dynamics within continuous optimal velocity models. Physical Review E 90, 042812 (2014). doi:10.1103/PhysRevE.90.042812

[32] Sugiyama, Y.: Optimal velocity model for traffic flow. Computer Physics Communications 121-122, 399-401 (1999). doi:10.1016/s0010-4655(99)00366-5

[33] Guckenheimer, J., Holmes, P.: Nonlinear oscillations, dynamical systems, and bifurcations of vector fields. Springer (1986)

[34] Stuart, A.M., Humphries, A.R.: Dynamical Systems and Numerical Analysis. Cambridge University Press, Cambridge (1996) 
[35] Nishinari, K., Kirchner, A., Namazi, A., Schadschneider, A.: Extended floor field ca model for evacuation dynamics. IEICE TRANSACTIONS on Information and Systems E87-D, 726-732 (2004)

[36] Kneidl, A., Borrmann, A., Hartmann, D.: Generation and use of sparse navigation graphs for microscopic pedestrian simulation models. Advanced Engineering Informatics 26(4), 669-680 (2012). doi:10.1016/j.aei.2012.03.006

[37] Kretz, T., Bönisch, C., Vortisch, P.: Comparison of various methods for the calculation of the distance potential field. In: Pedestrian and Evacuation Dynamics 2008, pp. 335-346. Springer Berlin Heidelberg (2009). doi:10.1007/978-3-642-04504-2_29

[38] Sethian, J.A.: A fast marching level set method for monotonically advancing fronts. Proceedings of the National Academy of Sciences 93(4), 1591-1595 (1996). doi:10.1073/pnas.93.4.1591

[39] Sethian, J.A.: Level Set Methods and Fast Marching Methods: Evolving Interfaces in Computational Geometry, Fluid Mechanics, Computer Vision, and Materials Science. Cambridge University Press, Cambridge (1999)

[40] Hartmann, D.: Adaptive pedestrian dynamics based on geodesics. New Journal of Physics 12,043032 (2010). doi: 10 .1088/1367-2630/12/4/043032

[41] Köster, G., Zönnchen, B.: Queuing at bottlenecks using a dynamic floor field for navigation. In: The Conference in Pedestrian and Evacuation Dynamics 2014, Transportation Research Procedia, pp. 344-352. Delft, The Netherlands (2014). doi:10.1016/j.trpro.2014.09.029

[42] Kretz, T.: Pedestrian traffic: on the quickest path. Journal of Statistical Mechanics: Theory and Experiment 2009(03), P03012 (2009). doi:10.1088/1742-5468/2009/03/P03012

[43] Bandini, S., Crociani, L., Vizzari, G.: Heterogeneous speed profiles in discrete models for pedestrian simulation. In: 93rd Transportation Research Board annual meeting, Washington, January 2014 - Committee number AHB45 - TRB Committee on Traffic Flow Theory and Characteristics (2014). URL http: / / arxiv.org/ abs/1401.8132

[44] von Sivers, I., Köster, G.: Dynamic stride length adaptation according to utility and personal space. Transportation Research Part B: Methodological 74, $104-117$ (2015). doi:10.1016/j.trb.2015.01.009

[45] Köster, G., Treml, F., Gödel, M.: Avoiding numerical pitfalls in social force models. Physical Review E 87(6), 063305 (2013). doi:10.1103/PhysRevE.87.063305 
[46] Robinson, S.: Simulation: The Practice of Model Development and Use. John Wiley $\&$ Sons (2004)

[47] Lewin, K.: Field theory in social science: Selected theoretical papers. Harper, New York (1951)

[48] Mcmullin, E.: What do physical models tell us? In: B.V. Rootselaar, J.F. Staal (eds.) Logic, Methodology and Philosophy of Science III, Studies in Logic and the Foundations of Mathematics, vol. 52, pp. 385-396. Elsevier (1968). doi:10.1016/S0049-237X(08)71206-0

[49] Gigerenzer, G., Todd, P.M., A.B.C. Research Group: Simple Heuristics That Make Us Smart. Oxford University Press, Oxford (1999)

[50] Moussaï, M., Nelson, J.D.: Simple heuristics and the modelling of crowd behaviours. In: U. Weidmann, U. Kirsch, M. Schreckenberg (eds.) Pedestrian and Evacuation Dynamics 2012, pp. 75-90. Springer International Publishing (2014). doi:10.1007/978-3-319-02447-9_5

[51] Köster, G., Seitz, M., Treml, F., Hartmann, D., Klein, W.: On modelling the influence of group formations in a crowd. Contemporary Social Science 6(3), 397-414 (2011). doi:10.1080/21582041.2011.619867

[52] Gigerenzer, G.: Why heuristics work. Perspectives on Psychological Science 3(1), 20-29 (2008). doi:10.1111/j.1745-6916.2008.00058.x 